\title{
OPtiCAL POLARIZATION OF 3C 265
}

\author{
M.H. COHEN, H.D. TRAN AND P.M. OGLE \\ California Institute of Technology \\ Pasadena, CA 91125 \\ AND \\ R.W. GOODRICH \\ Space Telescope Science Institute \\ Baltimore, MD 21218
}

\section{Spectropolarimetry, and a Quasar in 3C 265}

$3 \mathrm{C} 265$ is a high-redshift $(\mathrm{z}=0.811)$ radio galaxy showing extended emission line regions (EELR) to $50 \mathrm{kpc}$ from the nucleus (McCarthy et al 1995). However, it does not show the alignment effect (McCarthy 1993) that is common in distant galaxies: the EELR is not extended along the radio axis.

In December 1994 and January 1995 we observed 3C 265 with the spectropolarimeter at the $10-\mathrm{m}$ Keck Telescope. Figure 1 shows the total flux $\left(F_{\lambda}\right)$ and the polarized flux $\left(\mathrm{PF}_{\lambda}\right)$ of the nucleus. The $\mathrm{Mg}$ II $\lambda 2800$ doublet shows broad wings, as noted by Dey and Spinrad (1996). In polarized flux the equivalent width is $58 \pm 3 \AA$ (rest frame), which is within the normal range of radio-loud quasars (Wills et al 1995), and the quasar identification is strengthened by the velocity width, about $10,000 \mathrm{~km} \mathrm{sec}^{-1}(\mathrm{rms})$. By analogy to Seyfert galaxies we may say that $3 \mathrm{C} 265$ contains a quasar which is largely hidden by a dusty torus; we see its continuum and broad-line radiation by reflection (scattering). The equivalent width in $F_{\lambda}$ is less than in $P F_{\lambda}: E W\left(F_{\lambda}\right)=12 \pm 1 \AA$, which shows that the scattered quasar light is strongly diluted by starlight plus (probably) an unpolarized continuum (FC2) as in 3C 234 (Tran et al 1995). The narrow lines are all unpolarized, to within noise, so the NLR lies outside the torus and is ionized by photoionization or shock waves. 


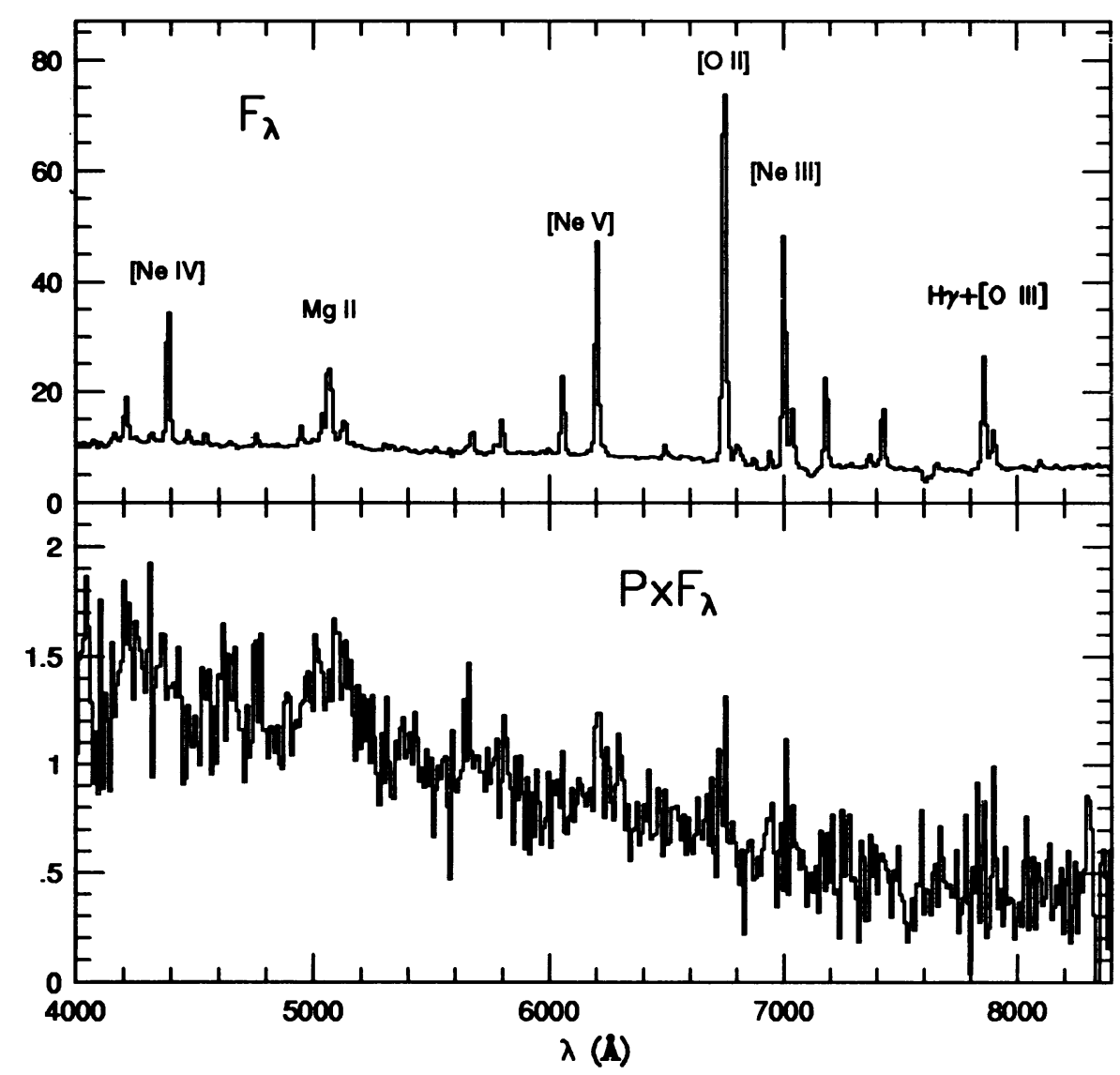

Figure 1. Top: 3C 265, Total flux of nucleus. Bottom: Polarized Flux. Note broad Mg II in emission in $P F_{\lambda}$, and that the narrow emission lines are unpolarized. The flux units are $10^{-18}$ erg cm $\mathrm{cm}^{-2} \mathrm{sec}^{-1} \AA^{-1}$.

\section{Imaging Polarimetry of 3C 265}

In May 1995 we made a polarization map of 3C 265 at Keck. The V-band filter passed $\mathrm{Mg}$ II $\lambda 2800$ and several weak narrow emission lines, but the image is dominated by the continuum. The effective resolution is $1.0^{\prime \prime}$. VLA images (Fernini et al 1993) show a lobe-dominated FR II source. The radio nucleus lies at the peak in optical intensity.

Figure 2 shows $3 \mathrm{C} 265$. The CCD pixels are $0.21^{\prime \prime}$ square and are binned 


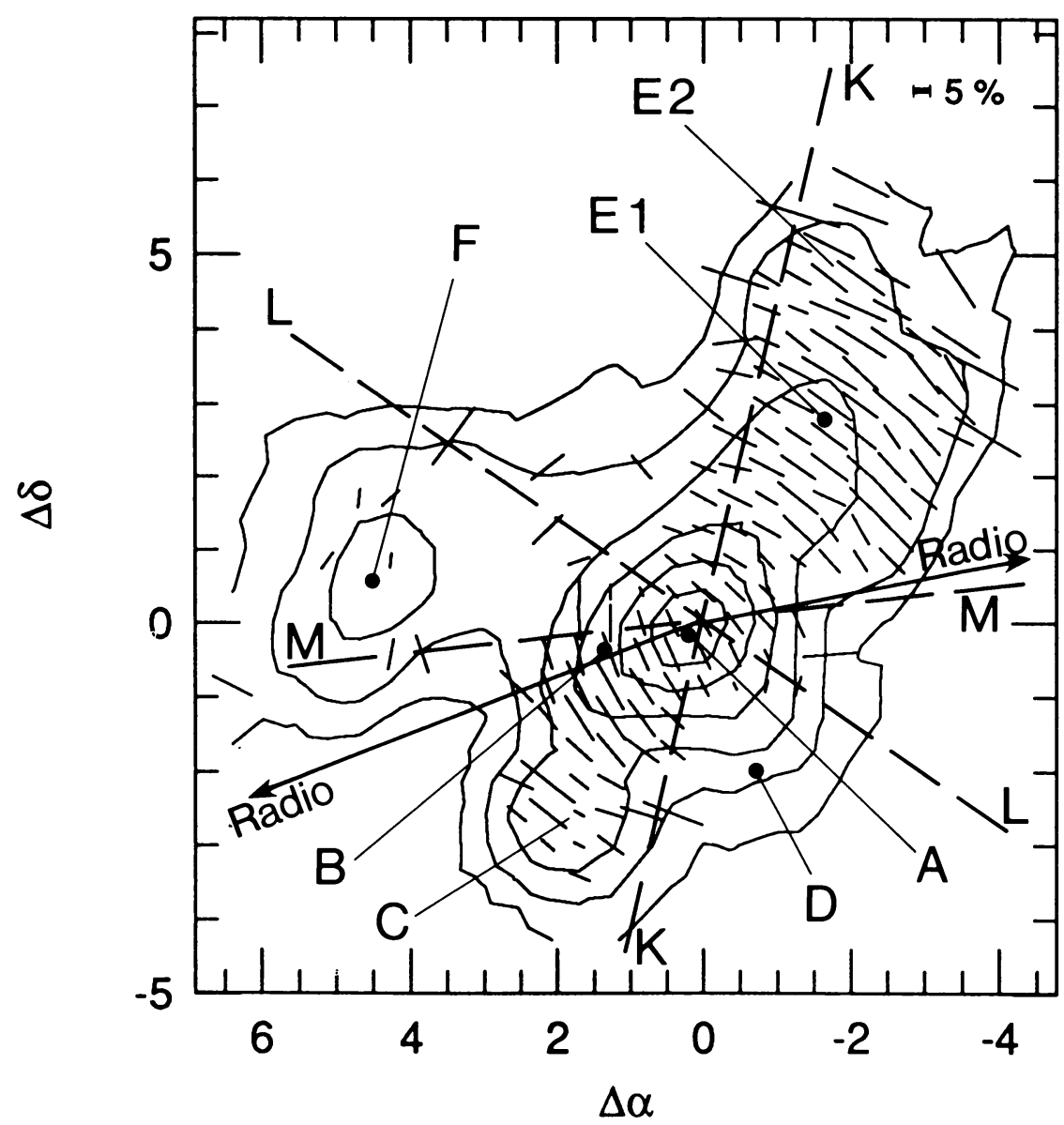

Figure 2. Polarization vectors superposed on intensity image of $3 \mathrm{C} \mathrm{265,} \mathrm{V} \mathrm{Band.} \mathrm{Scales}$ are in arc seconds. Contours are logarithmic in factors of 2 , starting at $2 \%$ of the peak. Radio axes are shown, and possible emission cones; see text.

$2 \times 2$ in Fig 2. The total exposure is $6480^{\text {s }}$, split into 4 settings of the waveplate. The polarization was calculated by averaging in flux and then combining the different waveplate settings. In Fig 2 only those vectors that exceed $2 \sigma$ in their bin are plotted. Table 1 shows the polarization in a few fields, each 2 bins square $\left(0.85^{\prime \prime}\right)$.

The vectors in Fig 2 (apart from the nucleus) are generally perpendicular to their nuclear radii, and the polarization must be produced by scattering of the nuclear light. 3C 265 is a gigantic reflection nebula. The 
TABLE 1. Polarization in $3 \mathrm{C} 265$

\begin{tabular}{crl}
\hline Region & P & $\theta$ \\
\hline A & $11 \%$ & $26^{\circ}$ \\
B & $18 \%$ & $31^{\circ}$ \\
C & $8 \%$ & $55^{\circ}$ \\
E1 & $15 \%$ & $54^{\circ}$ \\
E2 & $17 \%$ & $60^{\circ}$ \\
F & $2 \%$ & $-7^{\circ}$ \\
\hline
\end{tabular}

polarization is $11 \%$ at the nucleus (Region $A$ ) and increases to the southeast and north (Regions B, E). Regions D and F have much lower polarization, and the value for $F$ is only marginally significant. Region $F$ shows $O$ II $\lambda 3727$ emission at the same redshift as 3C 265 (McCarthy et al 1995) and might be an interacting galaxy. The foreground galaxy $12^{\prime \prime}$ to the east shows no polarization: $\mathrm{P}<1 \%(2 \sigma)$.

Section 1 invokes an obscuring torus, and we expect that there will be a cone of emission from it. Dashed lines KL in Fig 2 show a possible outline of the cone, with half-opening angle $55^{\circ}$ and axis along the $\mathrm{SE}$ radio axis at $111^{\circ}$. Regions $\mathrm{B} \mathrm{C}$ and $\mathrm{E}$ are in the cone and are strongly polarized. Region F may be weakly polarized by reflection since its mean vector is perpendicular to the radius. Region $D$ is at most weakly polarized and is largely out of the cone. An alternative cone of half angle $35^{\circ}, \mathrm{KM}$, catches most of the polarized regions and misses most of both D and F. However, this cone is not symmetric about the radio axis.

Our observations of 3C 265 are in accord with the emerging picture of powerful radio galaxies as quasars seen at high inclination. They also support the obscuring torus paradigm for radio galaxies in addition to Seyfert galaxies.

\section{References}

Dey, A. and Spinrad, H. (1996) The Radio Galaxy 3C265 Contains a Hidden Quasar Astroph. J., in press March 1996.

Fernini, I., Burns, J.O., Bridle, A.H. and Perley, R.A. (1993) Very Large Array Imaging of Five FR II 3CR Radio Galaxies, Astronom. J., Vol. no. 105, pp. 1690-1709.

McCarthy, P.J. (1993) High Redshift Radio Galaxies, Ann. Rev. Astron. and Astroph., Vol. no. 31, pp. 639-688.

McCarthy, P.J., Spinrad, H. and van Breugel, W. (1995) Emission-Line Imaging of 3CR Radio Galaxies. I. Imaging Data, Astroph. J. Supp., Vol. no. 99, pp. 27-66.

Tran, H.D., Cohen, M.H. and Goodrich R.W. (1995) Keck Spectropolarimetry of the Radio Galaxy 3C 234, A stronom. J., Vol. no. 110, in press December 1995.

Wills, B.J. et. al. (1995) The Hubble Space Telescope Sample of Radio-Loud Quasars: Ultraviolet Spectra of the First 31 Quaasrs, Astroph. J., Vol. no. 447, pp. 139-158. 